\title{
Elastocapillarity can control the formation and the morphology of beads-on-string structures in solid fibers
}

\author{
M. Taffetani ${ }^{1}$ and P. Ciarletta ${ }^{1,2, *}$ \\ ${ }^{1}$ MOX, Politecnico di Milano and Fondazione CEN-Centro Europeo di Nanomedicina, Piazza Leonardo da Vinci 32, 20133 Milan, Italy \\ ${ }^{2}$ CNRS and Sorbonne Universités, Université Paris 6, Institut Jean le Rond d'Alembert, UMR 7190, 4 place Jussieu case 162, \\ 75005 Paris, France
}

(Received 10 July 2014; published 23 March 2015)

\begin{abstract}
Beads-on-string patterns have been experimentally observed in solid cylinders for a wide range of material properties and structural lengths, from millimetric soft gels to nanometric hard fibers. In this work, we combine theoretical analysis and numerical tools to investigate the formation and nonlinear dynamics of such beaded structures. We show that this morphological transition is driven by elastocapillarity, i.e., a complex interplay between the effects of surface tension and bulk elasticity. Unlike buckling or wrinkling, the presence of an axial elongation is found here to favor the onset of fiber beading, in agreement with existing experimental results on electrospun fibers, hydrogels, and nerves. Our results also prove that the applied stretch can be used in fabrication techniques to control the morphology of the emerging beads-on-string patterns. Such quantitative predictions open the way for several applications, from tissue engineering to the design of stretchable electronics and the microfabrication of functionalized surfaces.
\end{abstract}

\section{INTRODUCTION}

Since the first experimental observation dating back to 1833 [1], it has been well known that the surface tension in a liquid filament can trigger the transition into a varicose shape, followed by a sudden breakup into droplets. Linear stability studies [2,3] have later proved that this phenomenon, thereafter named Rayleigh-Plateau instability (RPI), is governed by a competition between the surface tension, seeking to reduce the surface area at constant fluid volume, and the fluid inertia, opposing motions over long distances. More recent developments have focused on understanding the pearling in tubular membrane due to the competition between elastic bending and surface tension [4], propagation of fronts in RPI instability [5], nonlinear dynamics of the droplet formation in free-surface flows [6], and formation of blistering patterns during the capillary thinning of viscoelastic solutions [7]. Although elastic solids also possess a surface tension, the influence of a capillary effect for their macroscopic shaping can be often neglected, since the formation of surface patterns is contrasted by the high bulk energy required for an elastic deformation. Nevertheless, capillary and elastic forces scale down very differently while decreasing the typical size $L_{s}$ of the system [8], becoming comparable at low enough length scales. In fact, if $\mu$ is the shear modulus of the material and $\gamma$ is its surface tension, an elastocapillary interaction can be observed if the characteristic length $L_{e c}=\gamma / \mu$ is of the same order of $L_{s}$. Since $L_{e c}$ is in the submillimeter range [9], the ability of the surface tension to provoke a relevant deformation of a solid structure has been very recently observed in a number of miniaturized systems, such as coalescence in wet hair clumps [10], two-dimensional pattern formation in thin elastic films subjected to electrostatic forces or surface interactions $[11,12]$, creation of complex microscopic structures through

*pasquale.ciarletta@upmc.fr the wrapping of a liquid drop [13], or their pattern selection through a drop impact [14]. Moreover, an elastocapillary effect at more macroscopic scales might also occur for very soft solids, thus decreasing $\mu$ and increasing $L_{e c}$, as recently observed by immersing centimeter-scale elastic rods, made of a gel just above the percolation threshold, into an aqueous solution [15].

Similarly to the RPI, it is therefore expected that elastocapillary effects can drive the occurrence of a peristaltic pattern for elastic filaments with initial radius $R_{0}$ somewhat comparable to its capillary length $L_{e c}$. This morphological transition has been experimentally observed in applications spanning a wide range of elastic properties and characteristic lengths, from millimetric soft gel [16] to nanometric hard polymer fibers [17]. Although some theoretical analysis and empirical correlations have been recently proposed [18,19], the effects of elastocapillarity on the resulting beaded morphology in solids are barely understood. In particular, while it is well known that a compressive strain triggers many classical elastic instabilities, such as wrinkling [20] or buckling [21,22], bifurcations under tensile dead loads have been found only in elementary structures with at least one degree of freedom [23]. Thus, it is yet unclear why the axial elongation of a capillary elastic filament favors the onset of beading. This has been observed in few experiments with soft cylinders having very different physical and structural characteristics, including electrospun fibers [24], hydrogels [25], and nerve axons [26,27], as depicted in Fig. 1. In this perspective, this work aims at investigating how bulk elasticity and surface tension concur for driving a beading instability in soft elastic fibers, evaluating the onset conditions and its dynamics far from the bifurcation point. In particular, the main goal is to prove insights on the effects of the elastocapillarity on the onset and the nonlinear dynamics of the beaded patterns, opening new possibilities for generating controllable beads-on-string structures in a wide range of experimental applications. With this scope, both a theoretical analysis and numerical tools are 

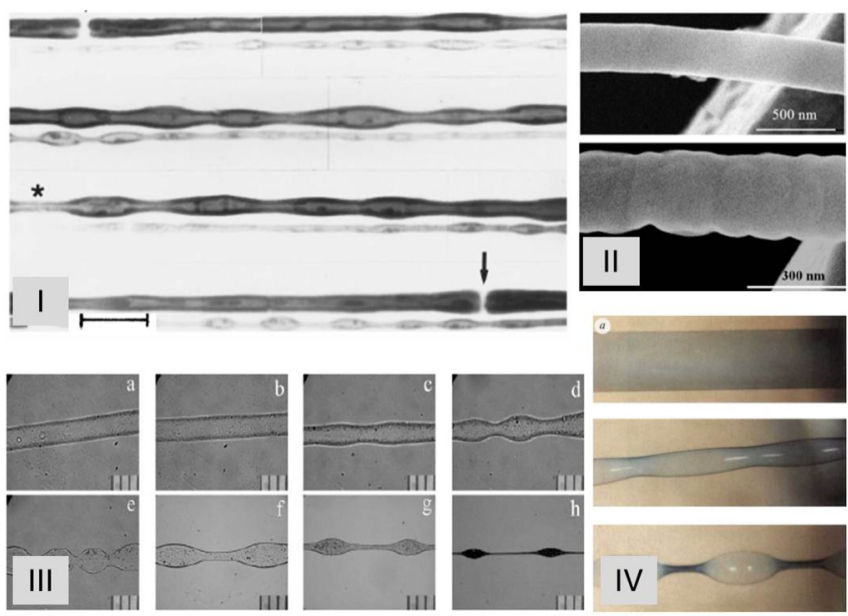

FIG. 1. (Color online) Bead-on-string patterns in solid fibers with different underlying physical properties: (I) rat sciatic nerve during a traction test (the scale bar is $50 \mu \mathrm{m}$, from Ref. [28]); (II) polymeric nanofiber under axial elongation (from Ref. [17]); (III) electrospun fiber during the extrusion process (the scale bar is 10 $\mu \mathrm{m}$, from Ref. [30]); (IV) stretched soft cylindrical gels with radius of few mm (from Ref. [25]).

proposed in the following to provide a quantitative prediction of the beaded morphology in the fully nonlinear regime.

\section{LINEAR STABILITY ANALYSIS}

Let us consider an elastic cylinder with radius $R_{0}$ and axial length $L_{0}$ subjected to an axial strain $\lambda_{z}$, so that $(r, z)$ and $(R, Z)$ are the polar coordinates in the spatial and material settings, respectively. Assuming that the filament is made of a neo-Hookean incompressible material with density $\rho$, three energy contributions can be taken into account: (i) the kinetic energy $K=(1 / 2) \int_{Z=0}^{L_{0}} d Z \int_{R=0}^{R_{0}} d R \rho \mathbf{v}^{2}$ where $\mathbf{v}$ is the spatial velocity; (ii) the bulk elastic energy $U_{e l}=$ $(\mu / 2) \int_{Z=0}^{L_{0}} d Z \int_{R=0}^{R_{0}} d R\left(J_{1}-3\right)$, where $J_{1}=\left[2+\sum_{j} u_{i, j}^{2}+\right.$ $\left.2 u_{i, i}+\left(1+u_{r} / R\right)^{2}\right], u_{i}$ indicates the component of the displacement along the direction $i$, with $(i, j)$ spanning over $(r, z)$, the comma denotes partial derivative, and the Einstein summation rule on dummy indices is considered; and (iii) the capillary energy on the free surface $U_{\text {surf }}=\gamma \int_{z=0}^{\lambda_{z} L_{0}} d z r \sqrt{1+r_{, z}^{2}}$. Using a variational approach, the equilibrium of the elastic filament arises from the minimization of the total energy by imposing a null variation of the Lagrangian functional $L$ as

$$
\delta L=\delta\left(K-U_{e l}-U_{\text {surf }}\right)=0 .
$$

The incompressibility constraint can be exactly fulfilled using a stream function $\phi=\phi(R, z, t)$ in a mixed coordinate state [18], so that $r^{2}=2 \phi_{, z}$ and $Z=(1 / R) \phi_{, R}$. Accordingly, the basic axis-symmetric solution after imposing the axial stretching $\lambda_{z}$ is given by $\phi_{0}(R, z, t)=(1 / 2) \lambda_{z} R^{2} z$. In order to perform a stability analysis of this static solution, let us consider a perturbation of the stream function as

$$
\phi(R, z, t)=\phi_{0}+\sum_{n=1}^{\infty} \epsilon^{n} R \phi_{n}(R, z, t),
$$

where $\epsilon$ is a small order parameter to be defined. Assuming variable separation, we look for a linear order solution in the form of a traveling wave with axial velocity $V$, being $\phi_{1}(R, z, t)=u^{(1)}\left(R / \sqrt{\lambda_{z}}\right) e^{I k(z-V t)}+$ c.c., where $I$ is the imaginary unit, c.c. indicates the complex conjugate, and $k=(2 \pi m) / L_{0}$ is the axial wave number of the perturbation, having integer mode $m$. Using Eq. (2), the bulk equilibrium from Eq. (1) reads

$$
\mathcal{L}_{1}\left[\mathcal{L}_{q}(u(r))\right]=0,
$$

where $\mathcal{L}_{p}=\partial_{r}^{2}+(1 / r) \partial_{r}-\left(1 / r^{2}\right)-\left(k^{2} p^{2}\right)$ for $p=(1, q)$, and $q=\sqrt{\lambda_{z}\left(\lambda_{z}^{2}-\frac{V^{2}}{c^{2}}\right)}$ with $c=\sqrt{\mu / \rho}$ being the speed of sound in the material.

Imposing regularity for $r=0$ and considering the boundary conditions $r_{0}=R_{0} / \sqrt{\lambda_{z}}$, the solution of Eq. (3) can be written as

$$
u^{(1)}(r)=\frac{I_{1}(k r q)}{I_{1}\left(k r_{0} q\right)}-\left(\frac{\lambda_{z}^{3}+1}{2}\right) \frac{I_{1}(k r)}{I_{1}\left(k r_{0}\right)},
$$

where $I_{n}$ is the modified Bessel function of order $n$. Moreover, the dispersion relation for a static critical wave with $V=0$ reads

$$
\begin{aligned}
& 2 \frac{\left(\lambda_{z}^{3}-1\right)}{\lambda_{z}^{\frac{3}{2}}}+\bar{k}\left[\frac{4}{\lambda_{z}^{\frac{1}{2}}} \frac{I_{0}\left(\bar{k} \lambda_{z}\right)}{I_{1}\left(\bar{k} \lambda_{z}\right)}-\frac{\left(\lambda_{z}^{3}+1\right)^{2}}{\lambda_{z}^{2}} \frac{I_{0}\left(\frac{\bar{k}}{\sqrt{\lambda_{z}}}\right)}{I_{1}\left(\frac{\bar{k}}{\sqrt{\lambda_{z}}}\right)}\right] \\
& +\frac{L_{e c}}{R_{o}}\left(\lambda_{z}^{3}-1\right)\left(1-\frac{\bar{k}^{2}}{\lambda_{z}}\right)=0,
\end{aligned}
$$

where $\bar{k}=k R_{0}$ is the dimensionless wave number. The marginal stability curves from Eq. (5) are depicted as lines in Fig. 2, showing the critical axial stretch $\lambda_{z}^{\text {th }}$ as a function of the elastocapillary ratio $L_{e c} / R_{0}$ for varying $\bar{k}$. In particular, it is found that the marginal stability curves superimpose for $\bar{k}<$ 0.01 , thus predicting the onset of a long wavelength beading. In absence of an axial strain, i.e., $\lambda_{z}=1$, the instability threshold is given by $L_{e c}=6 R_{0}$ [29]. Instead, if the cylinder is elongated, i.e., $\lambda_{z}>1$, the beading instability can occur at lower elastocapillary ratios $L_{e c} / R_{0}$ up to a minimum value given by $\left(L_{e c} / R_{0}\right)_{\min }=5.66$. This effect can be explained by a simple scaling argument. For the homogeneous solution the total surface energy reads $2 \pi \gamma R_{0} \sqrt{\lambda_{z}} L_{0}$, while the bulk elastic energy is given by $(\mu / 2) \pi R_{0}^{2} L_{0}\left(\lambda_{z}^{2}+2 \lambda_{z}^{-1}-3\right)$. Accordingly, the application of a small axial elongation provokes an increase of surface and elastic energies of order $\left(\lambda_{z}-1\right)$ and $\left(\lambda_{z}-1\right)^{2}$, respectively. Since the instability occurs when the capillary and the elastic contributions are of the same order, the axial stretch can favor the onset of beading at fixed elastocapillary ratio.

This theoretical result confirms the elastocapillary effect reported in the experimental observations of Fig. 1, giving analytical predictions for the required axial stretching for driving the onset of beading in an elastic filament with $L_{e c} / R_{0}>\left(L_{e c} / R_{0}\right)_{\min }$. Experimental data on the fabrication of nanofibers by electrospinning have evidenced this elastocapillary effect $[24,30]$; considering the typical surface tension in the range $\gamma=20-70 \mathrm{mN} / \mathrm{m}$ and a characteristic radius of $R_{0}=125 \mathrm{~nm}$, it has been shown that the formation of beads can be triggered by increasing the surface tension 

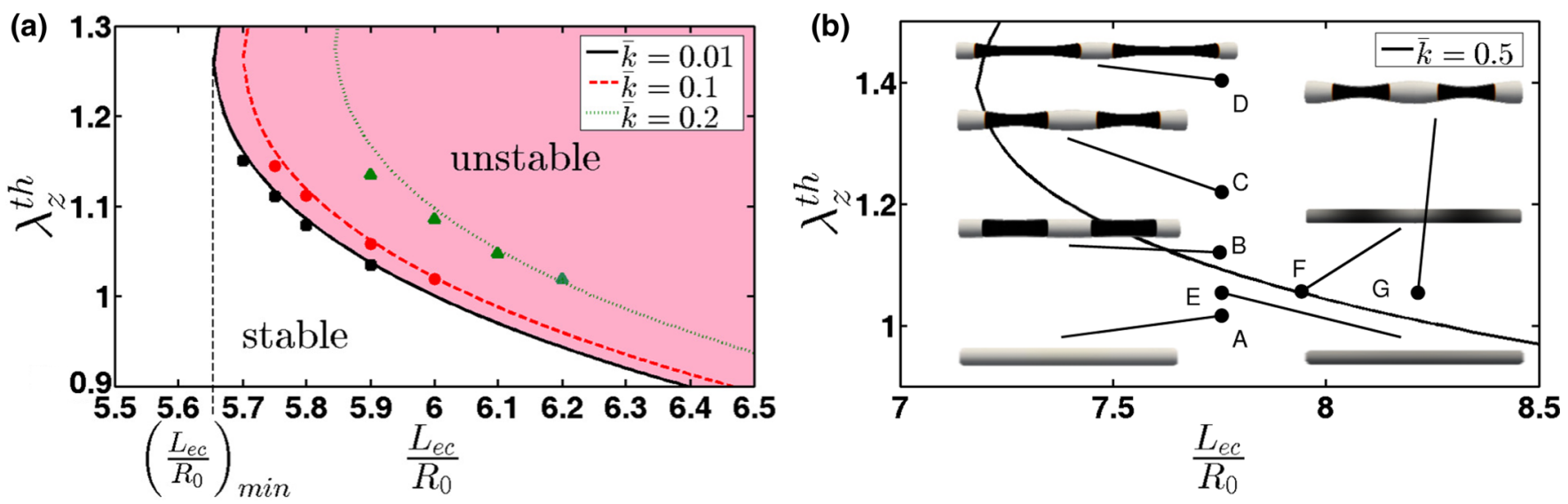

FIG. 2. (Color online) (a) Dispersion curves (solid black line, $\bar{k}=0.01$; dashed red line, $\bar{k}=0.1$; dotted green line, $\bar{k}=0.2$ ) showing the critical axial stretch $\lambda_{z}^{\text {th }}$ versus the elastocapillary ratio $L_{e c} / R_{0}$ at varying $\bar{k}$. The markers (black circles, $\bar{k}=0.01$; red squares, $\bar{k}=0.1$; green triangles, $\bar{k}=0.2$ ) indicate the thresholds computed numerically. (b) Morphological phase diagrams from the numerical simulations of two different paths, fixing $L_{e c} / R_{0}=7.7$ (A-B-C-D) and $\lambda_{z}=1.05$ (A-E-F-G).

(i.e., $L_{e c}$ ) above a critical value of about $\gamma=59.3 \mathrm{mN} / \mathrm{m}$ [24], which, from our theoretical prediction of $L_{e c}$ in the range [5.66-6] $R_{0}$, corresponds to a shear modulus of about $\mu=80-85 \mathrm{kPa}$, in agreement with the experimental measures [19]. The favoring effect of axial stretching on the formation of beading is also experimentally confirmed varying the applied electric field at fixed collecting distance reporting beading for lower elastocapillary ratios, i.e., $\left(L_{e c} / R_{0}\right)_{\min }<L_{e c} / R_{0}<6$, together with a morphological change from spindle- to spherelike beads [31,32].

\section{WEAKLY NONLINEAR ANALYSIS}

In order to study the dynamics of pattern formation of the beading instability, let us first perform a weakly nonlinear analysis using the multiple-scale method. Assuming a small increase of the axial stretch above the instability threshold, such that $\epsilon=\sqrt{\left(\lambda_{z}-\lambda_{z}^{t h}\right) / \lambda_{z}^{t h}} \ll 1$, the linear stability analysis predicts that the velocity of the near-critical wave is given by $V=\sqrt{3}\left(c \lambda_{z}^{t h}\right) \epsilon$. If we define a characteristic time $t_{c}=R_{0} / V$, the linear perturbation can be rewritten as $\phi_{1}(R, z, t)=A(\tau) u^{(1)}\left(R / \sqrt{\lambda_{z}}\right) e^{I k z}+$ c.c., where $\tau=\epsilon\left(t / t_{c}\right)$ is the slow time scale describing the growth of the amplitude $A(\tau)$ of the near-critical beads, which cannot be fixed by a linear analysis. In order to study the evolution of the beading beyond the instability threshold, we can extend the series development in Eq. (2) up to the third order in $\epsilon(n=3)$, taking into account the expression of the lower order terms to generate the resonating terms at higher orders. A solvability condition for the amplitude $A(\tau)$ can be obtained by considering that the total mechanical energy $E$ of the system must be conserved in absence of dissipative processes [33], so that

$$
\frac{d E}{d t}=\frac{d}{d t}\left[K+U_{e l}+U_{\text {surf }}\right]=0 .
$$

Imposing Eq. (6) at the fourth order in $\epsilon$ allows deriving the evolution law (also known as the Ginzburg-Landau equation) driving the growth of the beading amplitude in the weakly nonlinear regime, being

$$
\kappa \partial_{\tau}^{2} A(\tau)+v A(\tau)-\psi|A(\tau)|^{2} A(\tau)=0,
$$

where $\kappa$ and $v$ depend only on the linear order solution, $\psi$ is a complex function of the higher order terms, and the conjugate equation also applies. The amplitude Eq. (7) proves that the beading occurs after a pitchfork bifurcation: if $v / \psi>0$, there exists a stable solution $A^{s t}=\sqrt{\nu / \psi}$, so that the amplitude regularly grows as the square root of the distance from the critical stretch threshold, i.e., as $\epsilon B^{s t}$ with $B^{s t}=2 k \sqrt{\lambda_{z}} u\left(R_{0}\right) A^{s t}$ (supercritical bifurcation); if $v / \psi<0$, the pitchfork is unstable, and one expects that the solution has a discontinuity, jumping into a stable configuration which is driven by nonlinear effects of higher orders (subcritical bifurcation). In particular, it is found that the bifurcation turns supercritical when increasing both the critical wave number $\bar{k}^{t h}=2 \pi R_{0} / L_{0}$ and the critical axial stretch $\lambda_{z}^{t h}$ at the onset of the instability (see Supplemental Material [35] for further details). This means that the beading becomes continuous and controllable when applying a finite axial stretch to a solid cylinder with an aspect ratio $R_{0} / L_{0}$ beyond a given (small) value. Conversely, the instability can be weakly nonlinear unstable for very slender filaments: thus the analytical results predict a discontinuous beading formation just above the linear stability threshold, later controlled by nonlinearities.

\section{FULLY NONLINEAR NUMERICAL SIMULATIONS}

We have investigated the fully nonlinear dynamics of stretch-induced beading in solid cylinders implementing the elastocapillary problem on a finite element code. Numerical simulations are carried out by using the open source software FEniCS for solving partial differential equations [34]. To guarantee the incompressibility constraint, a mixed formulation with triangular Taylor-Hood elements is implemented, and the solution has been found through an incremental iterative Newton-Raphson method. The results of the numerical simulations are first validated versus the theoretical results of the linear stability analysis, as depicted in Fig. 2, where the numerical thresholds are indicated by the markers.

A morphological phase diagram from the simulations on the stretched cylinder is reported in Fig. 2(b), showing the nonlinear evolution of beading in different paths mimicking 


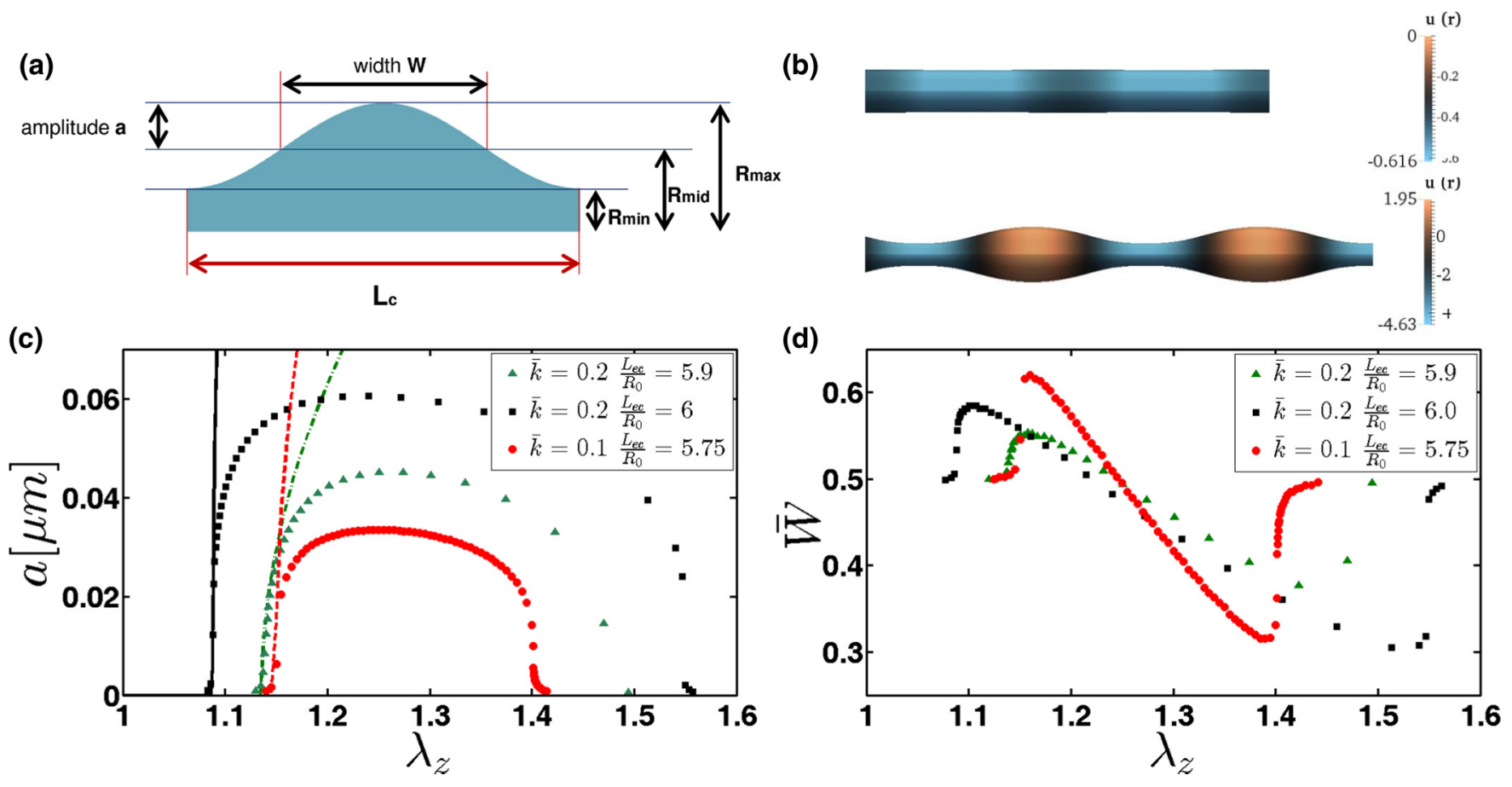

FIG. 3. (Color online) (a) Beading amplitude $a$ and axial width $W$. (b) Numerical simulation [35] mimicking the experiments on PAN nanofibers with $R_{0}=300 \mathrm{~nm}$ [17] giving (c) the amplitude $a$ and (d) the dimensionless width $\bar{W}=W / L_{c}$ for three different elastocapillary ratios. Numerical data (green triangles, $\bar{k}=0.2$ and $\frac{L_{e c}}{R_{0}}=5.9$; black squares, $\bar{k}=0.2$ and $\frac{L_{e c}}{R_{0}}=6$; red circles, $\bar{k}=0.1$ and $\frac{L_{e c}}{R_{0}}=5.75$ ) in (c) fit very well the theoretical predictions given by $\epsilon B^{s t}$ with no adjustable parameters: dot-dash green line, $S S E=1.59 \times 10^{-5}$; solid black line, $S S E=3.47 \times 10^{-3}$; dashed red line, $S S E=3.10 \times 10^{-4}$ (SSE: summed square of residuals).

two different experimental settings. The path (A-B-C-D) in Fig. 2(b) concerns the application of an axial elongation at constant elastocapillary ratio and corresponds to the stretching experiments shown in Fig. 1(I,II). The numerical results indicate a smooth growth of beads beyond the critical stretch $\lambda_{z}^{\text {th }}$, whose amplitudes later saturate to a constant value while their spacing increases with an increasing axial strain. This morphological transition corresponds to the experimental observations on nerve axons [see Fig. 1(I)], where the application of an axial stretch of about $10 \%$ triggers the formation of beaded structures evolving at almost fixed amplitude over a certain range of applied stress before disappearing. However, it must be noted that the use of a capillary energy is here a simplification of the elastic response of the outer membrane. Indeed, although a skin effect arises since the membrane thickness is much smaller than the radius of the nerve axon [26], a more accurate modeling of the membrane structure should include an elastic contribution in the corresponding surface energy.

The same behavior is observed by stretching polyacrylonitrile (PAN) nanofibers [see Fig. 1(II)], where ripples develop after a critical stretch of $\lambda_{z}^{\text {th }}=1.15$, which in our theoretical prediction gives an elastocapillary ratio of about $L_{e c} / R_{0}=5.75$.

Since PAN fibers are characterized by a surface tension $\gamma=0.025-0.1 \mathrm{~N} / \mathrm{m}$ and $R_{0}=300-600 \mathrm{~nm}$, such a prediction corresponds to a shear modulus in the range $\mu=6-60 \mathrm{kPa}$, which is consistent with the reported data [36]. Such experimental values of $\lambda_{z}^{\text {th }}$ and $L_{e c} / R_{0}$ have been used in numerical simulations in order to perform a quantitative comparison of the pattern dynamics. The resulting curves are depicted in Fig. 3, showing both the beading amplitude and their relative width versus the imposed stretch. In particular, ripples with amplitude of the order of magnitude of tents of nanometers are obtained, which correspond to the experimental observations [17]. Moreover, it is also confirmed that the beads' amplitude initially grows as the square root of the distance from the critical thresholds, as predicted by our weakly nonlinear analysis [see Fig. 3(c)]. Interestingly, while the amplitude is almost constant, the beading width decreases linearly with increasing axial stretch, as shown in Fig. 3(d). This is a very important finding, proving that the applied stretch can be used to control the resulting morphology of elastocapillary fibers in tensile experiments. Furthermore, increasing the applied stretch over a given threshold is found to ultimately drive the disappearance of the beads, due to the stabilizing effects enforced by the nonlinearity in the bulk elastic energy.

Conversely, the path (A-E-F-G) in Fig. 2(b) aims at mimicking the experiments of Matsuo and Tanaka [25], where dried cylindrical gels, with an initial radius $R_{0}$ in the range $0.35-0.5 \mathrm{~mm}$, are first stretched and then put in a water-acetone mixture, thus increasing the surface tension above the critical value for the elastocapillary ratio $L_{e c} / R_{0}$. A bubble pattern is there observed for critical axial elongations $\lambda_{z}^{\text {th }}$ up to $20 \%$, which in our theoretical analysis correspond to a gel shear modulus of tens of Pa for a surface tension of $\gamma=25 \mathrm{mN} / \mathrm{m}$ [37], consistent with recent experimental measures [15]. However, it is important to underline that it would be more accurate to use a diffuse interface energy (e.g., of the Ornstein-Zernicke type) to model such experiments, although a capillary energy 


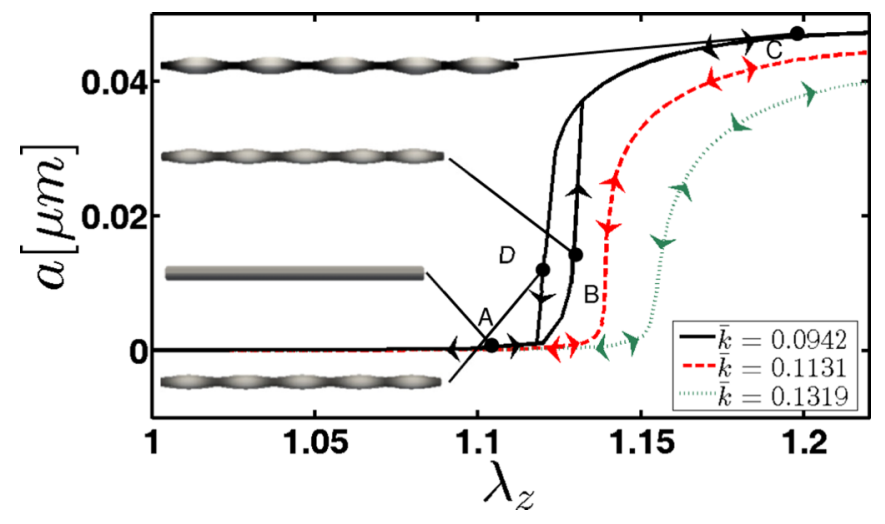

FIG. 4. (Color online) The beading amplitude $a$ versus the applied axial stretch in a loading-unloading cycle for different wave numbers $\bar{k}$ (solid black line, $\bar{k}=0.0942$; dashed red line, $\bar{k}=0.1131$; dotted green line, $\bar{k}=0.1319)$. We set $R_{0}=0.24 \mu \mathrm{m}, L_{0}=80 \mu \mathrm{m}$, $\mu=20 \mathrm{kPa}, \gamma=0.028 \mathrm{~N} / \mathrm{m}\left(L_{e c} / R_{0}=5.7\right)$.

is an acceptable modeling simplification when the correlation length is much smaller than the radius. The numerical results on the pattern dynamics are also qualitatively consistent with these experiments, reporting a bead change from spindlelike to spherelike while increasing the surface tension at fixed applied stretch. A continuous variation of the bead amplitude was also recently reported [16] in the absence of an axial strain, thus suggesting the occurrence of a supercritical bifurcation.

Nevertheless, the results of our weakly nonlinear analysis suggest that the beading instability is strongly dependent on the elastocapillary properties and on the geometry of the solid cylinder. In particular, the fully nonlinear simulations confirmed that the bifurcation can turn subcritical in very slender cylinders, as shown in Fig. 3. In this case, the beading amplitude for short-wavelength perturbations is found undergoing a sharp variation after the linear stability threshold, suddenly jumping into a finite value. Moreover, a hysteresis loop is observed if the applied stretch is gradually removed (path A-B-C-D in Fig. 4), although the instability is totally reversible. Both these features are indicative signs of a subcriticality of the elastocapillary instability, which is rather uncommon in elasticity, although a few examples have been recently investigated, such as sulcification [38], delamination [39], or fingering in a confined meniscus [40].

Although our theoretical predictions indicate that the wavelength of beading scales in general as the length $L_{0}$ of the

[1] F. Savart, Ann. Chim. Phys. 53, 337 (1883).

[2] J. Plateau, Experimental and Theoretical Statics of Liquids Subject to Molecular Forces Only (Gauthier-Villars, Paris, 1873).

[3] Lord Rayleigh, Proc. London Math. Soc. 10, 4 (1879).

[4] R. Bar-Ziv and E. Moses, Phys. Rev. Lett. 73, 1392 (1994).

[5] T. R. Powers and R. E. Goldstein, Phys. Rev. Lett. 78, 2555 (1997).

[6] J. Eggers, Rev. Mod. Phys. 69, 865 (1997).

[7] R. Sattler, C. Wagner, and J. Eggers, Phys. Rev. Lett. 100, 164502 (2008). solid cylinder, they also predict that shorter undulations have very close instability thresholds for very slender filaments. Accordingly, the wavelength selection might be driven by the presence of surface defects in the real practice, as found for wrinkling [41]. Thus, an imperfection-sensitivity analysis of beading should be performed in a future study. Further developments will also focus on the effect of other interaction potentials (e.g., electrostatic, magnetic, or intermolecular forces) on beading at lower scales of investigation.

\section{CONCLUSION}

In summary, we have presented both a theoretical and a numerical investigation of the elastocapillary effects driving beading instability in solid cylinders. Although our model does not claim describing all the underlying physical characteristics of the mentioned experiments, it proves that either the axial stretch $\lambda_{z}$ or the elastocapillary ratio $L_{e c}$ controls the beaded morphology over a large range of material properties and characteristic length scales. Proposing a weakly nonlinear analysis for a nonlinear elastic cylinder, quantitative predictions are given on the effects of the geometrical and the elastocapillary characteristics on the beading dynamics far from the bifurcation point. Thus, the results of this work can be used as guidelines for the fabrication of controllable patterns in a number of applications. Possible outcomes in material science concern the production of functional microthreads in biomaterials, e.g., for mimicking the beads-on-string spider web [42], as well as the design of nanowires with tunable properties for stretchable electronics [43] or for sensor devices in microelectromechanical systems [44]. Finally, the possibility to control the beading morphology opens new perspectives in many biological applications, from regenerative tissue engineering [45] to the fabrication of functionalized surfaces for cell culturing [46].

\section{ACKNOWLEDGMENTS}

This work was partially supported by the Start-up Packages and PhD Program project, cofunded by Regione Lombardia through the Fondo per lo sviluppo e la coesione 2007-2013, formerly FAS, and by the Progetto Giovani GNFM 2014, funded by the National Group of Mathematical Physics of the Istituto Nazionale di Alta Matematica (GNFM-INdAM).

[8] J. W. Van Honschoten, N. Brunets, and N. R. Tas, Chem. Soc. Rev. 39, 1096 (2010).

[9] B. Roman and J. Bico, J. Phys. Condens. Matter 22, 493101 (2010).

[10] J. Bico, B. Roman, L. Moulin, and A. Boudaoud, Nature (London) 432, 690 (2004).

[11] V. Shenoy and A. Sharma, Phys. Rev. Lett. 86, 119 (2001).

[12] A. Ghatak, M. K. Chaudhury, V. Shenoy, and A. Sharma, Phys. Rev. Lett. 85, 4329 (2000).

[13] C. Py, P. Reverdy, L. Doppler, J. Bico, B. Roman, and C. N. Baroud, Phys. Rev. Lett. 98, 156103 (2007). 
[14] A. Antkowiak, B. Audoly, C. Josserand, S. Neukirch, and M. Rivetti, Pub. Natl. Acad. Sci. USA 108, 10400 (2011).

[15] S. Mora, C. Maurini, T. Phou, J.-M. Fromental, B. Audoly, and Y. Pomeau, Phys. Rev. Lett. 111, 114301 (2013).

[16] S. Mora, T. Phou, J. M. Fromental, L. M. Pismen, and Y. Pomeau, Phys. Rev. Lett. 105, 214301 (2010).

[17] M. Naraghi, I. Chasiotis, H. Kahn, Y. Wen, and Y. Dzenis, Appl. Phys. Lett. 91, 151901 (2007).

[18] P. Ciarletta and M. Ben Amar, Soft Matter 8, 1760 (2012).

[19] J. H. Yu, S. V. Fridrikh, and G. C. Rutledge, Polymer 47, 4789 (2006).

[20] F. Brau et al., Nature Phys. 7, 56 (2011).

[21] K. Krieger, Nature (London) 488, 146 (2012).

[22] P. Ciarletta, Phys. Rev. Lett. 110, 158102 (2013).

[23] D. Zaccaria, D. Bigoni, G. Noselli, and D. Misseroni, Proc. R. Soc. A. 467, 1686 (2011).

[24] H. Fong, I. Chun, and D. H. Reneker, Polymer 40, 4585 (1999).

[25] F. S. Matsuo and T. Tanaka, Nature (London) 358, 482 (1992).

[26] S. Ochs, R. Pourmand, R. A. Jersild, and R. N. Friedman, Prog. Neurobiol. 52, 391 (1997).

[27] R. Pourmand, S. Ochs and R. A. Jersild Jr, Neuroscience 61, 373 (1994).

[28] V. S. Markin, D. L. Tanelian, R. A. Jersild Jr., and S. Ochs, Biophys. J. 76, 2852 (1999).

[29] B. Barriere, K. Sekimoto, and L. Leibler, J. Chem. Phys. 105, 1735 (1996).

[30] W. Zuo et al., Polym. Eng. Sci. 45, 704 (2005).

[31] J. M. Deitzel, J. Kleinmeyer, D. Harris and N. C. Beck Tan, Polymer 42, 261 (2001).
[32] S. Ramakrishna, K. Fujihara, W. E Teo, T. C. Lim, and Z. Ma, An Introduction to Electrospinning and Nanofibers (World Scientific, Singapore, 2005).

[33] Y. B. Fu and R. W. Ogden, Continuum Mech. Therm. 11, 141 (1999).

[34] A. Logg, K. A. Mardal, and G. N. Wells, Automated Solution of Differential Equations by the Finite Element Method (Springer, New York, 2012).

[35] See Supplemental Material at http://link.aps.org/supplemental/ 10.1103/PhysRevE.91.032413 for details on the theoretical derivation and video of the evolution of the pattern.

[36] X. F. Wu, Y. Y. Kostogorova-Beller, A. V. Goponenko, H. Hou, and Y. A. Dzenis, Phys. Rev. E 78, 061804 (2008).

[37] W. Shuhui and R. A. Shanks, J. Appl. Polym. Sci. 93, 1493 (2004).

[38] E. Hohlfeld and L. Mahadevan, Phys. Rev. Lett. 106, 105702 (2011).

[39] D. Vella, J. Bico, A. Boudaoud, B. Roman, and P. M. Reis, Pub. Natl. Acad. Sci. USA 106, 10901 (2009).

[40] J. S. Biggins, B. Saintyves, Z. Wei, E. Bouchaud, and L. Mahadevan, Pub. Natl. Acad. Sci. USA 110, 12545 (2013).

[41] Y. Cao and J. W. Hutchinson, Proc. R. Soc. A 468, 94 (2012).

[42] V. Sahni, D. V. Labhasetwar, and A. Dhinojwala, Langmuir 28, 2206 (2012).

[43] Y. Sun, W. M. Cho, H. Jiang, Y. Y. Huang, and J. A. Rogers, Nat. Nanotechnol. 1, 201 (2006).

[44] J. A. Rogers and Y. Huang, Pub. Natl. Acad. Sci. USA 106, 10875 (2009).

[45] P. Wallin et al., Biomicrofluidics 6, 024131 (2012).

[46] V. Beachley and X. Wen, Prog. Polym. Sci. 35, 868 (2010). 